\title{
Article
}

\section{Determination of Ankle and Metatarsophalangeal Stiffness During Walking and Jogging}

Mager, Fabian, Richards, James, Hennies, Malika, Dötzel, Eugen, Chohan, Ambreen, Mbuli, Alex and Capanni, Felix

Available at http://clok.uclan.ac.uk/23041/

Mager, Fabian, Richards, James ORCID: 0000-0002-4004-3115, Hennies, Malika, Dötzel, Eugen, Chohan, Ambreen ORCID: 0000-0003-0544-7832, Mbuli, Alex ORCID: 0000-0002-2104-5385 and Capanni, Felix (2018) Determination of Ankle and Metatarsophalangeal Stiffness During Walking and Jogging. Journal of Applied Biomechanics, 34 (6). pp. 448-453. ISSN 10658483

It is advisable to refer to the publisher's version if you intend to cite from the work. http://dx.doi.org/10.1123/jab.2017-0265

For more information about UCLan's research in this area go to

http://www.uclan.ac.uk/researchgroups/ and search for <name of research Group>.

For information about Research generally at UCLan please go to http://www.uclan.ac.uk/research/

All outputs in CLoK are protected by Intellectual Property Rights law, including Copyright law. Copyright, IPR and Moral Rights for the works on this site are retained by the individual authors and/or other copyright owners. Terms and conditions for use of this material are defined in the policies page.

\section{CLoK}

Central Lancashire online Knowledge www.clok.uclan.ac.uk

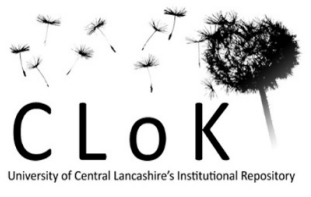


Determination of ankle and metatarsophalangeal stiffness during walking and jogging

Fabian Mager ${ }^{1}$, Jim Richards ${ }^{2}$, Malika Hennies ${ }^{1}$, Eugen Dötzel ${ }^{1}$, Ambreen Chohan ${ }^{2}$, Alex Mbuli $^{2}$, Felix Capanni ${ }^{1}$

${ }^{1}$ Faculty of Mechatronics and Medical Engineering, Ulm University of Applied Sciences, Germany

${ }^{2}$ Allied Health Research unit, University of Central Lancashire, UK

Conflict of Interest Disclosure: None.

\section{Correspondence Address:}

Professor Jim Richards

Professor of Biomechanics

Allied Health Research unit

University of Central Lancashire, Preston. UK

$+44(0) 1772894575$

jrichards@uclan.ac.uk

Running Title: Foot stiffness during walking and running 


\begin{abstract}
Forefoot stiffness has been shown to influence joint biomechanics. However, little or no data exists on metatarsophalangeal stiffness. Twenty-four healthy rearfoot strike runners were recruited from a staff and student population at the University of Central Lancashire. Five repetitions of shod, self-selected speed level walking and jogging were performed. Kinetic and kinematic data were collected using retro-reflective markers placed on the lower limb and foot, to create a three-segment foot model using the Calibrated Anatomical System Technique. Ankle and metatarsophalangeal moments and angles were calculated. Stiffness values were calculated using a linear best fit line of moment versus of angle plots. Paired t-tests were used to compare values between walking and jogging conditions. Significant differences were seen in ankle range of motion (ROM), but not in metatarsophalangeal ROM. Maximum moments were significantly greater in the ankle during jogging, but these were not significantly different at the metatarsophalangeal joint. Average ankle joint stiffness exhibited significantly lower stiffness when walking compared to jogging. However, the metatarsophalangeal joint exhibited significantly greater stiffness when walking compared to jogging. A greater understanding of forefoot stiffness may inform the development of footwear, prosthetic feet and orthotic devices, such as ankle-foot orthoses for walking and sporting activities.
\end{abstract}

Keywords: forefoot, ankle, joint stiffness, gait

Word Count: 2779 


\section{Introduction}

Forefoot stiffness has been shown to influence joint kinematics and kinetics. Willwacher et al. ${ }^{1}$ found that changes in the stiffness of the metatarsophalangeal joint have a bearing on power generation, push off duration and anterior shift of the centre of pressure (COP) in the second half of stance phase. Several studies have reported the vertical stiffness for the whole lower limb and joint stiffness for the ankle and knee joint during locomotion. ${ }^{3}$ These can be affected by different surfaces ${ }^{4}$ and footwear. ${ }^{5}$ In addition, an increase in knee joint stiffness and leg stiffness has been shown to increase with running speed, while ankle stiffness remains approximately constant. ${ }^{6}$ For other tasks, such as hopping, ankle stiffness has been identified as an important parameter. ${ }^{7}$ Sinclair et al. ${ }^{8}$ compared different running shoes and found that barefoot running leads to a higher leg and knee stiffness compared to running in conventional shoes. Whereas, Park et al. ${ }^{9}$ found no influence of a stiffened forefoot shoe on agility time and lower limb kinematics while playing badminton. However, these authors used a single segment foot model, which did not allow for the consideration of metatarsophalangeal joint angles and moments.

Kinematics of the ankle and metatarsophalangeal joint have been examined using multisegment foot models and it has been reported that, at $50 \%$ of the gait cycle the ankle joint is at peak dorsiflexion, with the metatarsophalangeal angle then moving to approximately $27^{\circ}$ of extension. ${ }^{2}$ To date, most studies have examined the stiffness properties and values of the ankle joint, knee joint and entire lower limb for different activities, but have omitted the metatarsophalangeal joint. Metatarsophalangeal angles, forces and moments during late stance may be measured using a three-segment foot model, which in turn could be used to determine metatarsophalangeal stiffness during late stance phase. However to the authors' knowledge, little or no data exists on metatarsophalangeal stiffness, despite this being important in facilitating the movement of the body over the foot during late stance phase. 
Previously reported outcome measures can be influenced by the different models and calculation methods. For example, linked segment body models combined with inverse dynamics are often used to calculate kinetics and kinematics of specific joints of the lower limb. Inverse dynamics often consider the foot as a single segment, and therefore not isolating the individual contributions of the calcaneal, metatarsal and phalangeal segments, but allowing a detailed analysis of the proximal joints to the foot.

Current literature recommends using a multi-segment foot model for walking and running, due to the individual segments of the foot having a significant influence on proximal joints. ${ }^{10,11}$ Previous studies have reported significant differences in ankle joint power generation when using a multi-segment foot, and this data has been used to form a simple spring-mass model to simulate the human leg. ${ }^{12}$ However, this only consists of one linear spring connected to body mass and therefore only considers one degree of freedom when describing overall stiffness of the leg. ${ }^{13}$ In addition stiffness may be described as linear stiffness, the change of elastic deformation, or the change of force divided by the elongation $\left[k_{x}=\Delta F / \Delta x\right]$, and torsional joint stiffness which may be defined as the change of moment divided by the change of angle $\left[k_{j}=\Delta M / \Delta \theta\right]$.

Torsional joint stiffness can be primarily linked to musculotendinous stiffness, with tendons being largely considered as passive components and muscles as active components. ${ }^{14}$ A certain level of stiffness is required to ensure stability, prevent soft tissue damage and store elastic energy within the musculoskeletal system. This concept is supported by previously reported data that too much stiffness increases loading rates due to a reduction in damping, which increases peak forces within the musculotendinous structures, resulting in increased risk of injury, such as stress fractures or osteoarthritis. However due to a lack of published data the direct relationship between stiffness and injury is not well understood ${ }^{15}$. 
Theoretically, optimizing mechanical properties such as mid and forefoot stiffness, may improve running economy. Increasing bending stiffness of running shoes has been shown to increase the lever arm around the metatarsophalangeal joint, due to anterior shift of the ground reaction force $(\mathrm{GRF}) .{ }^{1}$ Assuming the GRF remains the same, this would result in an increase of the metatarsal to phalangeal moment, however a clear understanding of the relationship between moment and stiffness of the metatarsophalangeal joint is lacking within the literature.

This study aimed to explore the differences in ankle and metatarsophalangeal joint stiffness during shod walking and jogging to provide a greater understanding of foot stiffness during these tasks. It was hypothesised that differences in ankle and metatarsophalangeal joint stiffness exist during shod walking and jogging.

\section{Methods}

Participants: Twenty-seven healthy subjects, of which twenty-four were rearfoot strikers, were recruited from a staff and student population at the University of Central Lancashire. The subjects consisted of 11 males and 16 females, with a mean age 25.6 years (SD 4.9), mean body weight 704.7 N (SD 118.3), and mean leg length $89.4 \mathrm{~cm}$ (SD 5.2). Exclusion criteria were any musculoskeletal injuries or disorders, history of surgery or traumatic injury of lower extremities, lower back pain, or any medical conditions that would limit physical activities. Written informed consent was obtained from all participants prior to study commencement. This study was conducted in accordance with the principles of the Declaration of Helsinki and was approved by the STEMH Ethics Committee of the University of Central Lancashire.

Procedures: Five repetitions of self-selected speed level walking and jogging were performed. Each subject was either wearing their own running shoe or was provided with a Winner Plus running shoe (Dr Comfort, USA). The same pair of shoes were worn for all trials. 
Kinetic data were collected at $200 \mathrm{~Hz}$ using four AMTI force plates and kinematic data were collected using a ten camera Oqus motion capture system (Qualisys medical AB, Sweden) at $100 \mathrm{~Hz}$. Passive retro-reflective markers were placed on the lower limbs using the Calibrated Anatomical System Technique to allow for segmental kinematics to be tracked in 6-degrees of freedom. ${ }^{16}$ In order to reduce measurement error, reflective markers were positioned by an experienced researcher and all data from each participant were collected during a single test session. ${ }^{17}$ Anatomical markers were positioned on the anterior superior iliac spine, posterior superior iliac spine, greater trochanter, medial and lateral femoral epicondyle, medial and lateral malleoli and over medial and lateral aspects of $1^{\text {st }}$ and $5^{\text {th }}$ metatarsal respectively. Additionally, clusters of non-collinear markers were attached to the shank and thigh. Markers were also placed over forefoot, mid-foot and rear foot aspects of the shoes (Figure 1).

Data analysis: Raw kinematic and kinetic data from the 24 rearfoot strikers were exported to Visual3D (C-Motion Inc., USA) software for analysis. Only rearfoot strikers were considered as this is a more common foot strike pattern (75-80\%) during endurance, slower speed running ${ }^{18}$. The rearfoot strikers were identified visually from the initial ground reaction force vector being under the heel in Visual3D. A three-segment foot model was used to calculate kinematic and kinetic data. Kinematic data were filtered using fourth order Butterworth filter with cut off frequency of $6 \mathrm{~Hz}$ and $15 \mathrm{~Hz}$ for walking and jogging respectively. Anatomical frames were defined by landmarks positioned at the medial and lateral borders of the joint, from these right-handed segment co-ordinate systems were defined. Joint kinematics were calculated based on the cardan sequence of $\mathrm{XYZ}$, equivalent to the joint coordinate system proposed by Grood and Suntay. ${ }^{19}$ Ankle and metatarsophalangeal joint kinetics were calculated using standard inverse dynamic methods, for the ankle relative to the shank coordinate system and for the metatarsophalangeal relative to the metatarsal coordinate system. Kinematic and kinetic data about the ankle and metatarsophalangeal were quantified 
from stance phase. The absolute ankle joint stiffness value was calculated from the slope of the moment versus joint angle graph using a best fit linear approach between the point where the moments become positive (dorsiflexion moments) to maximum dorsiflexion for both walking and jogging (Figure 2a, b, c, d). The metatarsophalangeal joint stiffness was calculated in a similar manner between the movement into extension to peak metatarsophalangeal moment for walking, and for the linear region between peak metatarsophalangeal moment to peak metatarsophalangeal angle for jogging (Figure 3a, b, c, d). These regions were chosen as they include the eccentric, stretching phase as the ankle moves into dorsiflexion and the metatarsophalangeal joint moves into extension. These phases are associated with the movement of the body over the stance limb and are likely to yield the greatest strain in the joint structures.

In addition, the shoe stiffness was calculated independently by placing markers on the Winner Plus running shoe using a technique similar to Oh and $\mathrm{Park}^{20}$. A three point bending stiffness test was performed with the shoe placed on two supporting frames, one of which was placed on the force plate. The forefoot of the shoe was then loaded 10 times with a vertical force, and the forefoot deflection angle and moment about the forefoot of the shoe were found. From these data the average bending stiffness was calculated from the slope of the moment/angle curve.

Statistical analysis: Descriptive statistics were used to calculate mean and standard deviations for each variable during walking and jogging conditions. Paired t-tests were used to compare ankle and metatarsophalangeal joint angles, moments and stiffness between walking and rearfoot strike jogging conditions. Statistical significance was set at $p<.05$, 


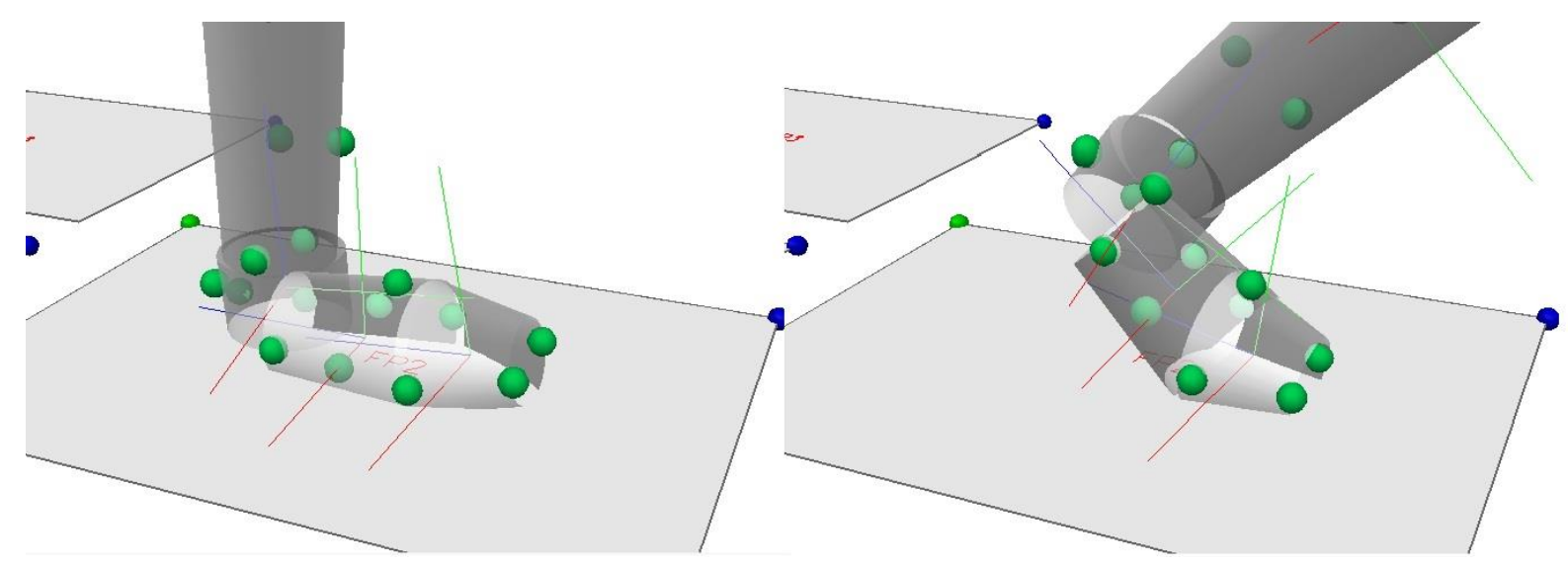

Figure 1.

\section{Results}

The means and standard deviations for the walking and jogging speeds were $1.45(0.15) \mathrm{m} / \mathrm{s}$ and $2.79(0.56) \mathrm{m} / \mathrm{s}$ respectively. Average ankle joint stiffness showed significantly lower stiffness when walking compared to jogging ( $p<0.001$ ), with values of 0.0110 (SD 0.0028) Nm.BW ${ }^{-1} \cdot \mathrm{deg}^{-1}$ and 0.0134 (SD 0.0028$) \quad \mathrm{Nm} . \mathrm{BW}^{-1} \cdot \mathrm{deg}^{-1}$ respectively. In contrast, the metatarsophalangeal joint showed significantly greater stiffness when walking compared to

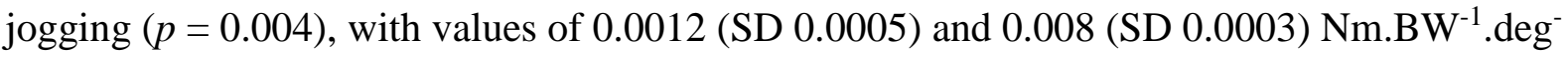
${ }^{1}$ respectively, equivalent of or 0.84 and $0.54 \mathrm{Nm} \mathrm{BW}^{-1}$ when not normalise to body weight (Table 1, Figure 2). Maximum moments increased significantly in the ankle $(p<0.001)$ between walking and jogging conditions. However, there were no significant differences in maximum moments at the metatarsophalangeal joint between walking and jogging $(p=0.623)$, (Table 2). Range of motion (ROM) throughout stance phase showed similar results to maximum moments, with significant differences seen in ankle ROM $(p<0.001)$ between walking and jogging. However, metatarsophalangeal ROM showed no significant difference ( $p=0.427$ ) between walking and jogging conditions, (Table 2). In addition, the testing of the shoe stiffness revealed a linear relationship between moment and angle, with an average extension angle of 23 degrees, which represents the typical angle seen during walking and running, and a peak moment of $1.6 \mathrm{Nm}$. The peak mean stiffness of the forefoot of the shoe 
was found to be 0.069 (SD 0.004 ) $\mathrm{Nm} / \mathrm{deg}$, accounting for only $8 \%$ and $13 \%$ of the metatarsophalangeal joint stiffness for walking and running respectively.

Table 1. Ankle and metatarsophalangeal (MTP) joint stiffness for walking and jogging, respectively.

\begin{tabular}{|c|c|c|c|c|c|}
\hline & \multicolumn{2}{|c|}{$\begin{array}{l}\text { Joint Stiffness } \\
\text { Nm.deg }\end{array}$} & \multicolumn{2}{|c|}{$\begin{array}{c}\text { Joint Stiffness / BW } \\
\left(\mathrm{Nm} \cdot \mathrm{BW}^{-1} \cdot \mathrm{deg}^{-1}\right)\end{array}$} \\
\hline & & Ankle & MTP & Ankle & MTP \\
\hline \multirow{2}{*}{ Walking } & Mean & 7.74 & 0.84 & 0.0110 & 0.0012 \\
\hline & SD & \pm 1.97 & \pm 0.32 & \pm 0.0028 & \pm 0.0005 \\
\hline \multirow{2}{*}{ Running } & Mean & 9.43 & 0.54 & 0.0134 & 0.0008 \\
\hline & SD & \pm 1.97 & \pm 0.22 & \pm 0.0028 & \pm 0.0003 \\
\hline \multirow{2}{*}{$\begin{array}{l}\text { Walking vs. } \\
\text { Running }\end{array}$} & Diff & & & 0.0024 & -0.0004 \\
\hline & P-Value & & & $<0.001$ & 0.002 \\
\hline
\end{tabular}

Joint Stiffness $\left(\mathrm{Nm} \cdot \mathrm{deg}^{-1}\right)$ and Joint Stiffness normalized to Body Weight $\left(\mathrm{Nm} \cdot \mathrm{BW} \mathrm{W}^{-1} \cdot \mathrm{deg}^{-1}\right)$. Significant differences marked in bold.

Table 2. Ankle and metatarsophalangeal (MTP) peak moments and range of motion for walking and running, respectively.

\begin{tabular}{|l|l|c|c|c|c|}
\hline \multicolumn{2}{|c|}{} & \multicolumn{2}{|c|}{$\begin{array}{c}\text { Max Moments } \\
\left(\mathrm{Nm} . \mathrm{BW}^{-1}\right)\end{array}$} & \multicolumn{2}{c|}{ ROM (deg) } \\
\cline { 3 - 6 } \multicolumn{2}{|c|}{} & Ankle & MTP & Ankle & MTP \\
\hline \multirow{3}{*}{ Walking } & Mean & 0.1617 & 0.0246 & 25.4493 & 22.4612 \\
\cline { 2 - 6 } & SD & \pm 0.0153 & \pm 0.0068 & \pm 3.4976 & \pm 4.9816 \\
\hline \multirow{3}{*}{ Running } & Mean & 0.2584 & 0.0251 & 43.2927 & 21.3533 \\
\cline { 2 - 6 } & SD & \pm 0.0363 & \pm 0.0078 & \pm 7.1112 & \pm 4.9632 \\
\hline $\begin{array}{l}\text { Walking vs. } \\
\text { Running }\end{array}$ & Diff & 0.0967 & 0.0004 & 17.8434 & -1.1079 \\
\cline { 2 - 6 } & P-Value & $<\mathbf{0 . 0 0 1}$ & 0.6229 & $<\mathbf{0 . 0 0 1}$ & 0.4270 \\
\hline
\end{tabular}

Peak moments normalized to BW and joint range of motion (ROM). Significant differences marked in bold. 
Figure 2.

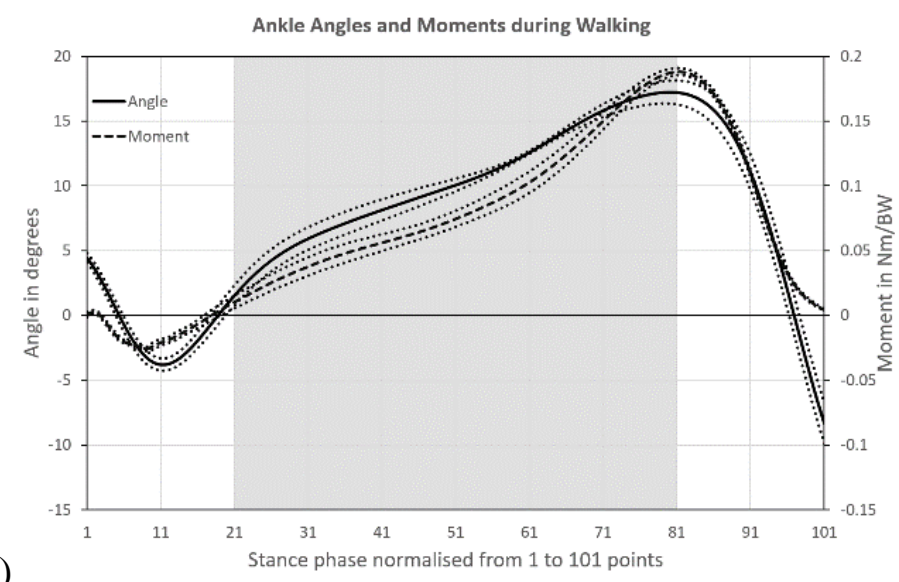

a)

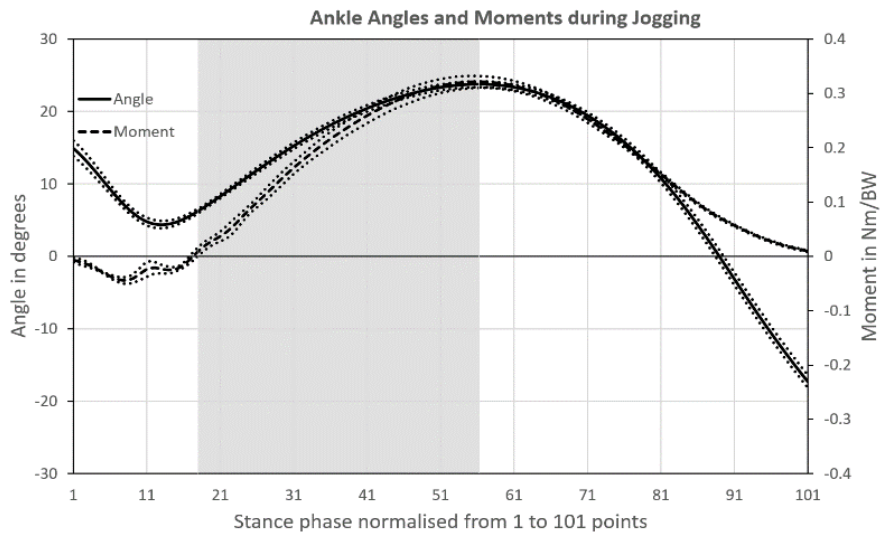

b)

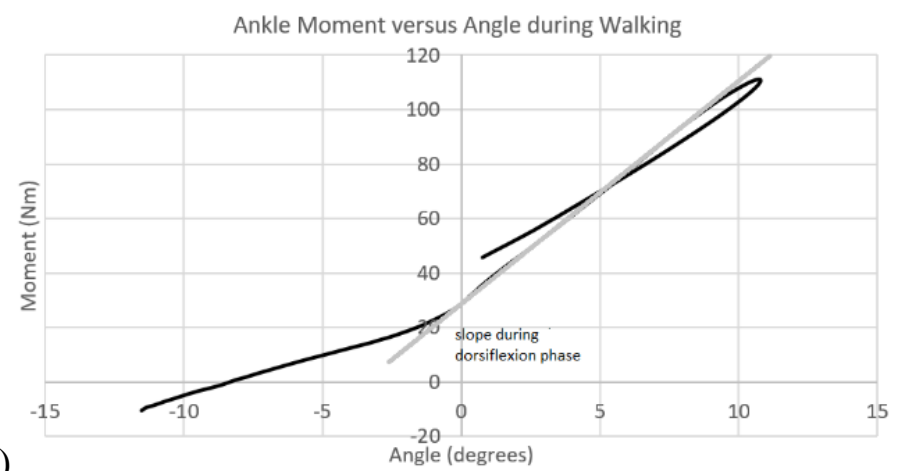

c)

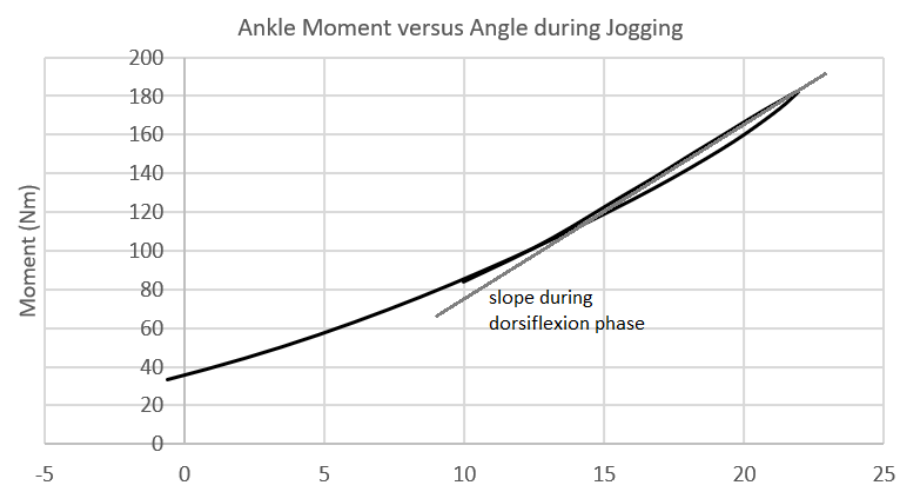

d)

Angle (degrees) 
Figure 3.

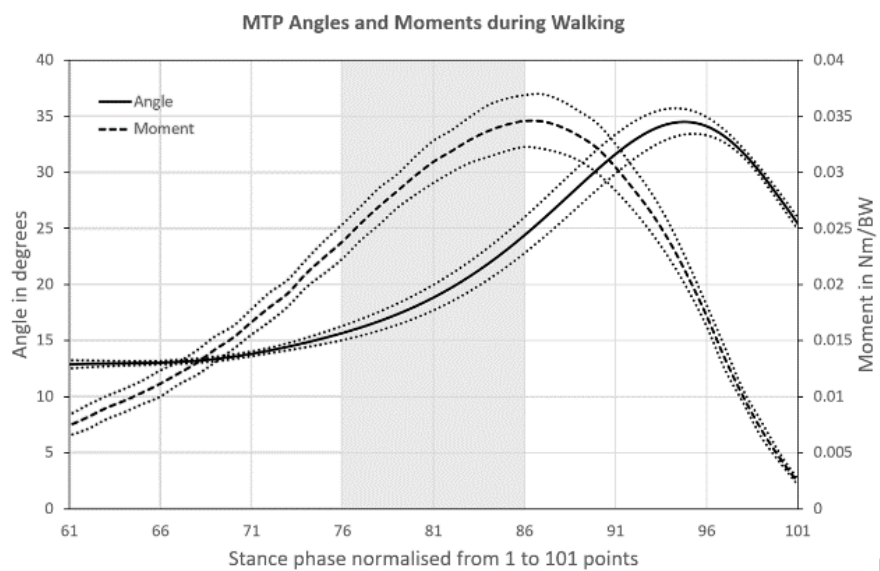

a)

MTP Angles and Moments during Jogging

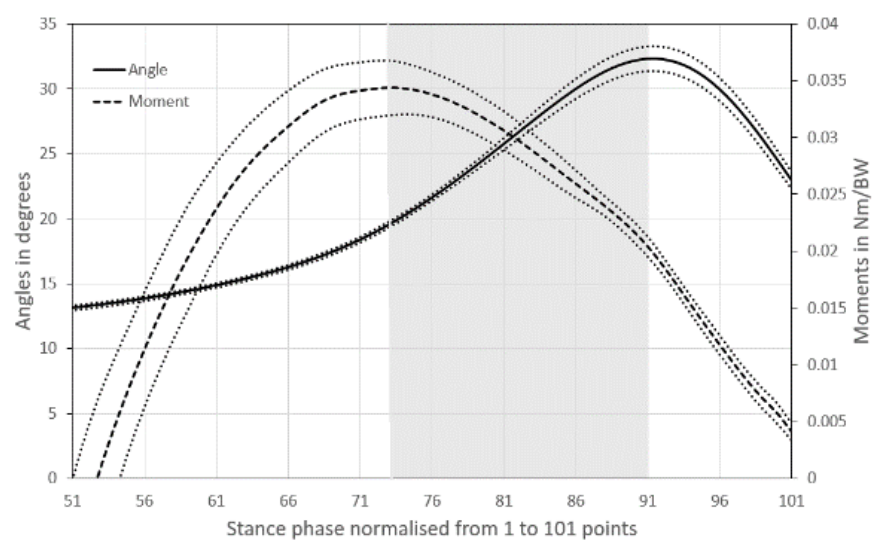

b)

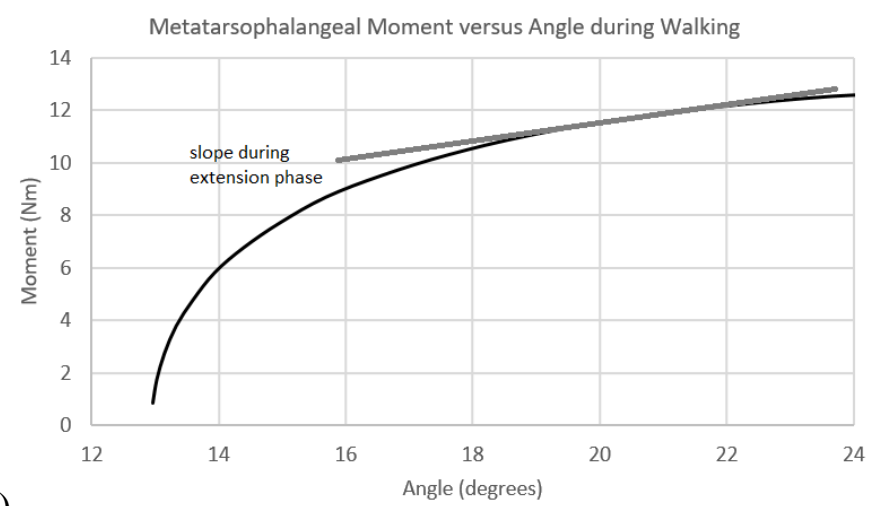

c)

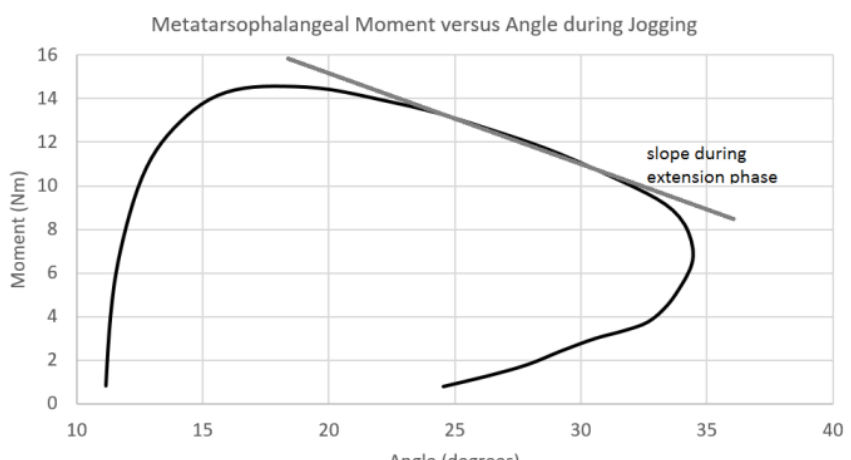

d)

Angle (degrees) 


\section{Discussion}

This study compared stiffness, maximum moments and joint angles in the ankle and metatarsophalangeal joint during shod walking and jogging. Walking speeds were similar to those previously reported Landry et $\mathrm{al}^{21}$ who reported a self-selected pace of $1.38 \mathrm{~ms}-1( \pm 0.19)$ in their healthy control group, and running speed was at the upper range of slow jogging $(1.5 \mathrm{~m} / \mathrm{s}$ to $3 \mathrm{~m} / \mathrm{s}$ ) reported by Keller et $\mathrm{al}^{22}$. At these speeds it was found that ankle stiffness during jogging was significantly higher than in walking $(p<.001)$. This is in agreement with Apps et al., ${ }^{5}$ who found that during loading phase of walking ankle stiffness was $0.0066 \mathrm{Nm} . \mathrm{BW}^{-1} . \mathrm{deg}^{-}$ ${ }^{1}$ compared to $0.0128 \mathrm{Nm} \cdot \mathrm{BW}^{-1} \cdot \mathrm{deg}^{-1}$ during jogging. Different outcomes can be primarily explained by considering the different parts of the stance phase. Apps et al..$^{5}$ considered the first and last third of stance phase for walking and the first and last half, of stance phase for jogging to calculate ankle stiffness. Kuitunen et al. ${ }^{6}$ found that ankle stiffness remains the same with increasing jogging speed $\left(7 \mathrm{Nm} \cdot \mathrm{deg}^{-1}\right)$, where ankle stiffness was calculated for the braking phase only. Reduced to average BW, this would mean a stiffness value of 0.0099 Nm.BW ${ }^{-1} \cdot \mathrm{deg}^{-1}$. Hobara et al. ${ }^{23}$ also found constant ankle stiffness on the sound leg of amputees with increasing jogging speed $\left(0.017 \mathrm{Nm} \cdot \mathrm{BW}^{-1} \cdot \mathrm{deg}^{-1}\right)$, where ankle stiffness was calculated in the middle of stance phase. Different calculation methods make it difficult to compare stiffness values between studies, highlighting the importance of standardised methods for calculating stiffness. Bearing these differences in mind, the values found in the current study are similar to previous studies. The ankle stiffness calculation used in this study is limited by using only positive moments to maximum dorsiflexion (Figure $2 \mathrm{a}$ and $\mathrm{b}$ ), as the influence of nearby joints can be neglected, and stiffness values are therefore largely constant over time.

The authors could find no published values in literature for metatarsophalangeal joint stiffness. This is therefore the first known study to suggest stiffness values for the metatarsophalangeal joint. The calculated values for metatarsophalangeal joint stiffness during 
shod walking and jogging were 0.0012 and $0.0008 \mathrm{Nm} . \mathrm{BW}^{-1} \cdot \mathrm{deg}^{-1}$, respectively, with a significant decrease of $0.0004 \mathrm{Nm} \cdot \mathrm{BW}^{-1} \cdot \operatorname{deg}^{-1}(p<0.001)$ observed between conditions. These stiffness values were calculated during the final phase of push off (Figure 3a and b).

No significant differences were found in the maximum moment around the metatarsophalangeal joint for walking and jogging conditions $\left(0.0246\right.$ and $0.0251 \mathrm{Nm} . \mathrm{BW}^{-1}$, respectively). Olesen et al. ${ }^{24}$ compared metatarsophalangeal stiffness for barefoot running against running shoe bending stiffness and found maximum moments of about $0.0431 \mathrm{Nm} . \mathrm{BW}^{-}$ 1 .

Ankle and metatarsophalangeal joint ROM values from the current study are largely in accordance with values found in previous research. ${ }^{2,25}$ As expected, significant differences were found for ankle joint ROM between walking and jogging conditions. Although a change in stiffness doesn't necessarily correlate with a change in the maximum moment and the ROM, these parameters will have an effect on the moment against angle curve. As stiffness is the slope of the moment against angle curve, a change of moments or angular deflection is expected. However, in the current study significant differences were found only for maximum ankle moment and ankle ROM, but not for the metatarsophalangeal joint. Therefore, maximum moment and ROM values do not appear to be decisive parameters for measuring these changes. Since metatarsophalangeal stiffness was calculated during the movement into metatarsophalangeal extension these parameters do not appear to have a direct influence on stiffness. Differences in stiffness during walking and jogging conditions may be caused by faster COP movement over metatarsophalangeal segments. This would change the slope of moment and angular deflection; however, this may not necessarily influence peak moment and deflection. Further investigation is required to confirm this theory.

The stiffness values reported in this study may have applications for the development of mechanical properties in the manufacturing of prosthetic feet and prescription of ankle foot 
orthosis (AFO). Currently, it is not known whether the mechanical stiffness properties of such devices ensure optimal walking and running ability. For example, the forefoot design of AFOs is largely dependent on the experience of the orthotist and is currently not based on an objective assessment of forefoot stiffness. Stiffness will have major influence on gait parameters, such as energy cost and return and the function in pathological gait. Bregmann et al. ${ }^{26}$ developed a device to determine AFO characteristics such as neutral angles, ankle and metatarsophalangeal joint stiffness. The stiffness values suggested in the current study may inform the design of prostheses and orthoses, which more accurately match optimal characteristics.

The current study has shown that ankle and metatarsophalangeal joint stiffness are significantly altered in walking compared to jogging. This finding would suggest that different types of prosthesis or orthoses are required depending on the activity and performance level of a patient. For amputees, stability and security will have a major influence on proprioception of the optimal stiffness value, which may be higher than the values suggested in this study. Therefore, further work is required to determine whether stiffness values from sound feet are appropriate for determining optimal stiffness values for prostheses and orthoses. In addition, further work could consider the interaction between metatarsophalangeal and ankle joint stiffness in the optimization of designs of orthotic/prosthetic components.

There are limitations to the calculation of dynamic metatarsophalangeal stiffness used in this study, as this is the stiffness of both the joint and the shoe. Further independent testing on the Winner Plus running shoe indicated that the shoe stiffness accounted for $8 \%$ and $13 \%$ of the metatarsophalangeal joint stiffness for walking and running respectively, however there may be further interactions between the shoe and the foot which remain unexplored. The Winner Plus running shoe was representative of the shoes used within this study, however differences in shoe stiffness between makes and models of running shoes may exist ${ }^{20}$. In addition, the foot may have moved within the shoe, changing the position of the foot relative 
to the marker positions affecting the apparent lever arm and joint moment, therefore further work is required to support these initial findings.

\section{Acknowledgement}

None declared 


\section{References}

1. Willwacher S, König M, Braunstein B, Goldmann J, Brüggemann G. The gearing function of running shoe longitudinal bending stiffness. Gait Posture. 2014;40(3):386-390.

2. Leardini A, Benedetti MG, Catani F, Simoncini L, Giannini S. An anatomically based protocol for the description of foot segment kinematics during gait. Clin Biomech. 1999; 14:528-536.

3. Michael G, Blickhan R. Joint stiffness of the ankle and the knee in running. J Biomech. 2002;35:1459-1474.

4. Farley CT, Houdijk HH, Van Strien C, Louie M. Mechanism of leg stiffness adjustment for hopping on surfaces of different stiffnesses. J Appl Physiol. 1998;85(3):1044-1055.

5. Apps C, Sterzing T, Brien TO, Lake M. Lower limb joint stiffness and muscle cocontraction adaptations to instability footwear during locomotion. J Electromyogr Kinesiol. 2016;31:55-62.

6. Kuitunen S, Komi P V, Kyröläinen H. Knee and ankle joint stiffness in sprint running. Med Sci Sports Exerc. 2002;34(1):166-173.

7. Farley CT, Morgenroth DC. Leg stiffness primarily depends on ankle stiffness during human hopping. J Biomech. 1999;32:267-273.

8. Sinclair J, Richards J, Taylor PJ, Shore H. The influence of semi-custom orthoses on multi-segment foot kinematics in males. Foot Ankle Online J. 2015;1941-6806.

9. Park S, Lam W, Yoon S, et al. Effects of forefoot bending stiffness of badminton shoes on agility, comfort perception and lower leg kinematics during typical badminton movements. Sport Biomech. 2017;1476-3141:1-13.

10. Bezodis N. Modeling the Stance Leg in Two-Dimensional Analyses of Sprinting: Inclusion of the MTP Joint Affects Joint Kinetics. J Appl Biomech. 2012;28:222-227.

11. Bruening DA, Cooney KM, Buczek FL. Gait \& Posture Analysis of a kinetic multisegment foot model part II : Kinetics and clinical implications. Gait Posture. 2012;35(4):535540 .

12. Blickhan R. The spring-mass model for running and hopping. $\mathrm{J}$ Biomech. 1989;22(11):1217-1227.

13. Latash ML, Zatsiorsky M. Joint stiffness: Myth or reality? Hum Mov Sci. 1993; 12:653-692.

14. Kelly LA, Lichtwark G, Cresswell AG, Cresswell AG. Active regulation of longitudinal arch compression and recoil during walking and running. J R Soc Interface. 2014;12(102):20141076.

15. Butler RJ, Crowell HP, Davis IM. Lower extremity stiffness : implications for performance and injury. Clin Biomech. 2003;18:511-517.

16. Cappozzol A, Catan F, Crocel U Della, Leardini A. Position and orientation in space of bones during movement: anatomical frame definition and determination. Clin Biomech. 1995;10(4):171-178.

17. Yu B, Kienbacher T, Growney ES, Johnson ME, An K. Reproducibility of the Kinematics and Kinetics of the Lower Extremity during Normal Stair-Climbing. J Orthop Res. 1997:348-352.

18. Hasegawa H., Yamauchi T, Kraemer WJ. Foot strike patterns of runners at $15 \mathrm{~km}$ point during an elite-level half marathon. J. Strength Cond. Res. 2007;21,888-893.

19. Grood E, Suntay W. A joint coordinate system for the clinical description of threedimensional motions: application to the knee. Trans ASME. 1983;105:136-144.

20. Oh K., Park S. The bending stiffness of shoes is beneficial to running energetics if it does not disturb the natural MTP joint flexion. Journal of biomechanics. 2017;28(53),127-135 
21. Landry, S.C., McKean, K.A., Hubley-Kozey, C.L., Stanish, W.D. and Deluzio, K.J. Knee biomechanics of moderate OA patients measured during gait at a self-selected and fast walking speed. Journal of biomechanics. 2007;40(8),1754-1761.

22. Keller, T.S., Weisberger, A.M., Ray, J.L., Hasan, S.S., Shiavi, R.G. and Spengler, D.M. Relationship between vertical ground reaction force and speed during walking, slow jogging, and running. Clinical biomechanics, 1996;11(5),253-259.

23. Hobara H, Baum BS, Kwon HJ, et al. Amputee locomotion: Spring-like leg behavior and stiffness regulation using running-specific prostheses. J Biomech. 2013;46(14):2483-2489. 24. Oleson M, Adler D, A PG. A comparison of forefoot stiffness in running and running shoe bending stiffness. J Biomech. 2005;38:1886-1894.

25. Novacheck TF. The biomechanics of running. Gait Posture. 1998;7:77-95.

26. Bregman DJJ, Rozumalski A, Koops D, Groot V De, Schwartz M, Harlaar J. Runner Up of the ESMAC 2008 Best Paper Award A new method for evaluating ankle foot orthosis characteristics : BRUCE. Gait Posture. 2009;30:144-11 
